Supporting Information for

\title{
MULTICENTER BONDING IN CARBOCATIONS WITH TETRACOORDINATE PROTONS
}

\author{
Robert Ponec* \\ Institute of Chemical Process Fundamentals, Czech Academy of Sciences, \\ Prague 6, Suchdol 2, 165 02, Czech Republic \\ Patrick Bultinck* \\ Department of Inorganic and Physical Chemistry, Ghent University, \\ Krijgslaan 281, B-9000 Gent, Belgium \\ Pradeep Gutta and Dean J. Tantillo* \\ Department of Chemistry, University of California, Davis, One Shields \\ Avenue, Davis, CA 95616, USA
}




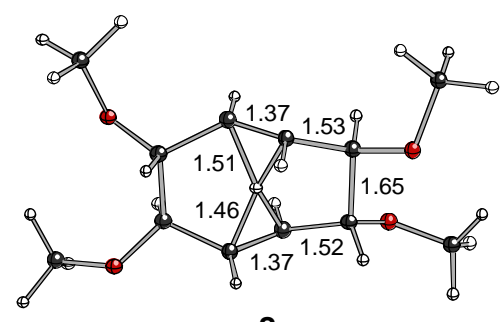

8

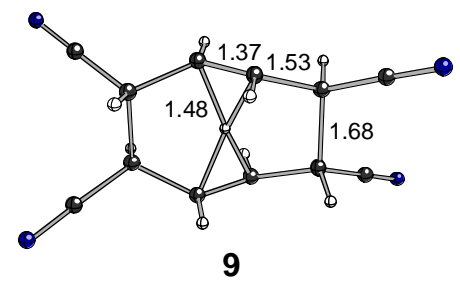

9

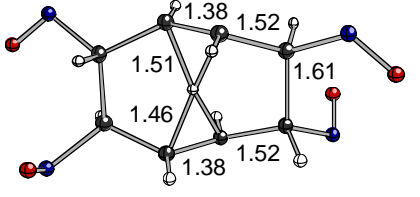

10

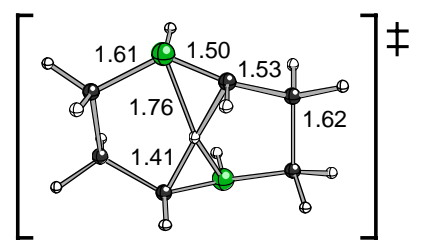

11
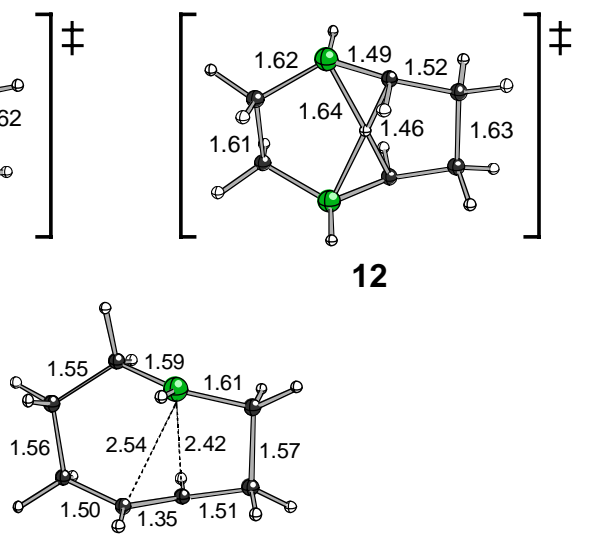

13

8

\begin{tabular}{|c|c|c|c|c|c|}
\hline \multicolumn{6}{|c|}{$\begin{array}{l}\mathrm{B} 3 \mathrm{LYP} / 6-31+\mathrm{G}(\mathrm{d}, \mathrm{p}) \\
\mathrm{E}=-770.4078204 \mathrm{a} . \mathrm{u} . \\
\mathrm{ZP} \text { correction: } 0.314226 \mathrm{a} \cdot \mathrm{u} . \\
0 \text { imaginaries }\end{array}$} \\
\hline \multirow{2}{*}{$\begin{array}{l}\text { Center } \\
\text { Number }\end{array}$} & \multirow{2}{*}{$\begin{array}{l}\text { Atomic } \\
\text { Number }\end{array}$} & Atomic & \multicolumn{3}{|c|}{ Coordinates (Angstroms) } \\
\hline & & Type & $\mathrm{X}$ & $\mathrm{Y}$ & Z \\
\hline 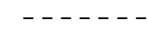 & & $-x_{0}$ & -------1 & -------- & ------- \\
\hline 1 & 6 & 0 & 1.927389 & 0.678608 & -0.093065 \\
\hline 2 & 1 & 0 & 2.083558 & 0.901337 & -1.160284 \\
\hline 3 & 6 & 0 & 1.929566 & -0.968539 & 0.020330 \\
\hline 4 & 6 & 0 & 0.549705 & -1.438591 & -0.408299 \\
\hline 5 & 6 & 0 & -0.549886 & -1.439275 & 0.409223 \\
\hline 6 & 6 & 0 & -1.929197 & -0.968515 & -0.019862 \\
\hline 7 & 1 & 0 & -2.132755 & -1.195834 & -1.076159 \\
\hline 8 & 6 & 0 & -1.926789 & 0.679155 & 0.092914 \\
\hline 9 & 1 & 0 & -2.082996 & 0.901972 & 1.160093 \\
\hline 10 & 6 & 0 & -0.566284 & 1.189564 & -0.386679 \\
\hline 11 & 6 & 0 & 0.566400 & 1.188789 & 0.386227 \\
\hline 12 & 1 & 0 & -0.402920 & -1.455140 & 1.492087 \\
\hline 13 & 1 & 0 & 2.133220 & -1.195785 & 1.076646 \\
\hline 14 & 1 & 0 & 0.000570 & -0.153205 & 0.000654 \\
\hline 15 & 1 & 0 & 0.454561 & 1.196683 & 1.474548 \\
\hline 16 & 1 & 0 & -0.454694 & 1.198154 & -1.475003 \\
\hline 17 & 1 & 0 & 0.402313 & -1.452987 & -1.491144 \\
\hline 18 & 8 & 0 & 2.878355 & -1.505917 & -0.841871 \\
\hline 19 & 8 & 0 & -2.878821 & -1.504519 & 0.842239 \\
\hline 20 & 8 & 0 & -2.994877 & 1.098173 & -0.698447 \\
\hline 21 & 8 & 0 & 2.995129 & 1.098441 & 0.698318 \\
\hline 22 & 6 & 0 & 4.188142 & -1.743629 & -0.290545 \\
\hline 23 & 1 & 0 & 4.120814 & -2.414473 & 0.573975 \\
\hline 24 & 1 & 0 & 4.755291 & -2.228801 & -1.084524 \\
\hline 25 & 1 & 0 & 4.670312 & -0.807144 & -0.000493 \\
\hline 26 & 6 & 0 & -4.187659 & -1.744340 & 0.289594 \\
\hline 27 & 1 & 0 & -4.755995 & -2.227025 & 1.084244 \\
\hline 28 & 1 & 0 & -4.669575 & -0.808912 & -0.004263 \\
\hline 29 & 1 & 0 & -4.118765 & -2.418051 & -0.572572 \\
\hline
\end{tabular}




\begin{tabular}{|c|c|c|c|c|c|}
\hline 30 & 6 & 0 & -3.422635 & 2.446167 & -0.453192 \\
\hline 31 & 1 & 0 & -2.618249 & 3.160367 & -0.671987 \\
\hline 32 & 1 & 0 & -4.260698 & 2.627935 & -1.125658 \\
\hline 33 & 1 & 0 & -3.749784 & 2.566604 & 0.586705 \\
\hline 34 & 6 & 0 & 3.421675 & 2.446861 & 0.453312 \\
\hline 35 & 1 & 0 & 2.616735 & 3.160313 & 0.672511 \\
\hline 36 & 1 & 0 & 4.259757 & 2.629165 & 1.125608 \\
\hline 37 & 1 & 0 & 3.748448 & 2.567868 & -0.586639 \\
\hline
\end{tabular}

9

B3LYP / 6-31+G $(d, p)$

$E=-681.1871578 \mathrm{a} . \mathrm{u}$.

ZP correction: 0.178831 a.u.

0 imaginaries

\begin{tabular}{|c|c|c|c|c|c|}
\hline \multirow{2}{*}{$\begin{array}{l}\text { Center } \\
\text { Number }\end{array}$} & \multirow{2}{*}{$\begin{array}{l}\text { Atomic } \\
\text { Number }\end{array}$} & \multirow{2}{*}{$\begin{array}{l}\text { Atomic } \\
\text { Type }\end{array}$} & \multicolumn{3}{|c|}{ Coordinates (Angstroms) } \\
\hline & & & $\mathrm{X}$ & $\mathrm{Y}$ & $\mathrm{Z}$ \\
\hline 1 & 6 & 0 & -1.922723 & 0.836031 & 0.093629 \\
\hline 2 & 1 & 0 & -2.058713 & 1.036419 & 1.162449 \\
\hline 3 & 6 & 0 & -1.922723 & -0.836031 & -0.093629 \\
\hline 4 & 6 & 0 & -0.559650 & -1.312975 & 0.396573 \\
\hline 5 & 6 & 0 & 0.559650 & -1.312975 & -0.396573 \\
\hline 6 & 6 & 0 & 1.922723 & -0.836031 & 0.093629 \\
\hline 7 & 1 & 0 & 2.058713 & -1.036419 & 1.162449 \\
\hline 8 & 6 & 0 & 1.922723 & 0.836031 & -0.093629 \\
\hline 9 & 1 & 0 & 2.058713 & 1.036419 & -1.162449 \\
\hline 10 & 6 & 0 & 0.559650 & 1.312975 & 0.396573 \\
\hline 11 & 6 & 0 & -0.559650 & 1.312975 & -0.396573 \\
\hline 12 & 1 & 0 & 0.435045 & -1.333219 & -1.481789 \\
\hline 13 & 1 & 0 & -2.058713 & -1.036419 & -1.162449 \\
\hline 14 & 1 & 0 & 0.000000 & 0.000000 & 0.000000 \\
\hline 15 & 1 & 0 & -0.435045 & 1.333219 & -1.481789 \\
\hline 16 & 1 & 0 & 0.435045 & 1.333219 & 1.481789 \\
\hline 17 & 1 & 0 & -0.435045 & -1.333219 & 1.481789 \\
\hline 18 & 6 & 0 & -3.033512 & 1.399874 & -0.664577 \\
\hline 19 & 7 & 0 & -3.900346 & 1.837257 & -1.299579 \\
\hline 20 & 6 & 0 & -3.033512 & -1.399874 & 0.664577 \\
\hline 21 & 7 & 0 & -3.900346 & -1.837257 & 1.299579 \\
\hline 22 & 6 & 0 & 3.033512 & -1.399874 & -0.664577 \\
\hline 23 & 7 & 0 & 3.900346 & -1.837257 & -1.299579 \\
\hline 24 & 6 & 0 & 3.033512 & 1.399874 & 0.664577 \\
\hline 25 & 7 & 0 & 3.900346 & 1.837257 & 1.299579 \\
\hline
\end{tabular}

10

B3LYP / 6-31G (d)

$E=-829.3659685$ a.u.

ZP correction: 0.169284 a.u.

0 imaginaries

\begin{tabular}{|c|c|c|c|c|c|}
\hline \multirow{2}{*}{$\begin{array}{l}\text { Center } \\
\text { Number }\end{array}$} & Atomic & Atomic & \multicolumn{3}{|c|}{ Coordinates (Angstroms) } \\
\hline & Number & Type & $\mathrm{X}$ & $\mathrm{Y}$ & $\mathrm{Z}$ \\
\hline 1 & 6 & 0 & -1.911063 & 0.786851 & 0.208217 \\
\hline 2 & 1 & 0 & -2.072819 & 1.067215 & 1.251915 \\
\hline 3 & 6 & 0 & -1.907167 & -0.819117 & 0.057987 \\
\hline 4 & 6 & 0 & -0.533756 & -1.347438 & 0.428102 \\
\hline 5 & 6 & 0 & 0.534051 & -1.344080 & -0.438310 \\
\hline 6 & 6 & 0 & 1.907245 & -0.818048 & -0.064004 \\
\hline 7 & 1 & 0 & 2.196396 & -1.063895 & 0.968494 \\
\hline 8 & 6 & 0 & 1.910923 & 0.788977 & -0.202485 \\
\hline 9 & 1 & 0 & 2.072472 & 1.076968 & -1.244129 \\
\hline 10 & 6 & 0 & 0.590189 & 1.276773 & 0.361163 \\
\hline 11 & 6 & 0 & -0.590352 & 1.279257 & -0.351738 \\
\hline 12 & 1 & 0 & 0.326170 & -1.366602 & -1.511361 \\
\hline
\end{tabular}




\begin{tabular}{|c|c|c|c|c|c|}
\hline 13 & 1 & 0 & -2.196609 & -1.057454 & -0.976192 \\
\hline 14 & 1 & 0 & -0.000196 & -0.004944 & -0.000993 \\
\hline 15 & 1 & 0 & -0.537929 & 1.292114 & -1.443777 \\
\hline 16 & 1 & 0 & 0.537733 & 1.280497 & 1.453276 \\
\hline 17 & 1 & 0 & -0.325669 & -1.377197 & 1.500940 \\
\hline 18 & 7 & 0 & -3.073306 & 1.416865 & -0.595953 \\
\hline 19 & 7 & 0 & -2.985047 & -1.445869 & 0.934222 \\
\hline 20 & 7 & 0 & 3.073216 & 1.413126 & 0.606223 \\
\hline 21 & 7 & 0 & 2.985603 & -1.438092 & -0.944437 \\
\hline 22 & 8 & 0 & -3.233343 & 0.889429 & -1.659661 \\
\hline 23 & 8 & 0 & -3.870634 & -0.688657 & 1.207682 \\
\hline 24 & 8 & 0 & 3.232911 & 0.878226 & 1.666257 \\
\hline 25 & 8 & 0 & 3.870964 & -0.678614 & -1.212247 \\
\hline
\end{tabular}

\section{1}

\begin{tabular}{|c|c|c|c|c|c|}
\hline & & & ------ & - - - - - - & \\
\hline Center & Atomic & Atomic & Coor & inates (An & roms) \\
\hline Number & Number & Type & $\mathrm{X}$ & $\mathrm{Y}$ & Z \\
\hline 1 & 6 & 0 & 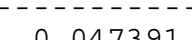 & 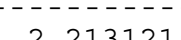 & 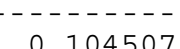 \\
\hline $\begin{array}{l}1 \\
2\end{array}$ & 6 & 0 & -1.402164 & 1.527163 & -0.119067 \\
\hline 3 & 6 & 0 & -1.402164 & 0.085751 & 0.387219 \\
\hline 4 & 6 & 0 & -0.047391 & -2.213121 & 0.104507 \\
\hline 5 & 6 & 0 & 1.402164 & -1.527163 & -0.119067 \\
\hline 6 & 1 & 0 & -1.205645 & -1.051490 & -1.646718 \\
\hline 7 & 1 & 0 & 0.000000 & 0.000000 & 0.319263 \\
\hline 8 & 1 & 0 & 1.205645 & 1.051490 & -1.646718 \\
\hline 9 & 1 & 0 & 1.412351 & -0.017035 & 1.482973 \\
\hline 10 & 1 & 0 & -1.412351 & 0.017035 & 1.482973 \\
\hline 11 & 1 & 0 & 0.049985 & 3.185341 & -0.410062 \\
\hline 12 & 1 & 0 & -2.158084 & 2.160014 & 0.369901 \\
\hline 13 & 1 & 0 & 2.158084 & -2.160014 & 0.369901 \\
\hline 14 & 1 & 0 & -0.049985 & -3.185341 & -0.410062 \\
\hline 15 & 1 & 0 & -1.616696 & 1.545030 & -1.196512 \\
\hline 16 & 1 & 0 & 1.616696 & -1.545030 & -1.196512 \\
\hline 17 & 1 & 0 & 0.148749 & 2.414006 & 1.183730 \\
\hline 18 & 1 & 0 & -0.148749 & -2.414006 & 1.183730 \\
\hline 19 & 5 & 0 & -1.109987 & -1.130595 & -0.435781 \\
\hline 20 & 5 & 0 & 1.109987 & 1.130595 & -0.435781 \\
\hline 21 & 6 & 0 & 1.402164 & -0.085751 & 0.387219 \\
\hline
\end{tabular}

\section{2}

B3LYP $/ 6-31+G(d, p)$

$E=-286.1385383$ a.u

ZP correction: $0.173273 \mathrm{a} . \mathrm{u}$.

1 imaginary@-774 $\mathrm{cm}^{-1}$

\begin{tabular}{|c|c|c|c|c|c|}
\hline Center & Atomic & Atomic & \multicolumn{3}{|c|}{ Coordinates (Angstroms) } \\
\hline Number & Number & Type & $\mathrm{X}$ & $\mathrm{Y}$ & Z \\
\hline 1 & 6 & 0 & 0.590291 & 0.563136 & 1.970548 \\
\hline 2 & 6 & 0 & -0.590291 & -0.563136 & 1.970548 \\
\hline 3 & 6 & 0 & -0.590291 & -1.236430 & 0.609729 \\
\hline 4 & 6 & 0 & -0.427132 & -0.679792 & -2.031959 \\
\hline 5 & 6 & 0 & 0.427132 & 0.679792 & -2.031959 \\
\hline 6 & 6 & 0 & 0.590291 & 1.236430 & 0.609729 \\
\hline 7 & 1 & 0 & -2.232098 & -0.030940 & -0.551692 \\
\hline 8 & 1 & 0 & 0.000000 & 0.000000 & 0.119606 \\
\hline 9 & 1 & 0 & -0.300303 & 1.867578 & 0.481530 \\
\hline 10 & 1 & 0 & 2.232098 & 0.030940 & -0.551692 \\
\hline
\end{tabular}




\begin{tabular}{|c|c|c|c|c|c|}
\hline 11 & 1 & 0 & 0.300303 & -1.867578 & 0.481530 \\
\hline 12 & 1 & 0 & 0.418700 & 1.249510 & 2.811612 \\
\hline 13 & 1 & 0 & -0.418700 & -1.249510 & 2.811612 \\
\hline 14 & 1 & 0 & 1.057951 & 0.698949 & -2.934758 \\
\hline 15 & 1 & 0 & -1.057951 & -0.698949 & -2.934758 \\
\hline 16 & 1 & 0 & -1.543598 & -0.045469 & 2.152014 \\
\hline 17 & 1 & 0 & -0.280440 & 1.519986 & -2.122332 \\
\hline 18 & 1 & 0 & 1.543598 & 0.045469 & 2.152014 \\
\hline 19 & 1 & 0 & 0.280440 & -1.519986 & -2.122332 \\
\hline 20 & 5 & 0 & -1.250856 & -0.747171 & -0.637216 \\
\hline 21 & 5 & 0 & 1.250856 & 0.747171 & -0.637216 \\
\hline
\end{tabular}

\section{3}

B3LYP $/ 6-31+G(d, p)$

$E=-299.415852$ a.u.

ZP correction: 0.186539 a.u.

0 imaginaries

\begin{tabular}{|c|c|c|c|c|c|}
\hline \multirow{2}{*}{$\begin{array}{l}\text { Center } \\
\text { Number }\end{array}$} & \multirow{2}{*}{$\begin{array}{l}\text { Atomic } \\
\text { Number }\end{array}$} & \multirow{2}{*}{$\begin{array}{c}\text { Atomic } \\
\text { Type }\end{array}$} & \multicolumn{3}{|c|}{ Coordinates (Angstroms) } \\
\hline & & & $\mathrm{X}$ & $\mathrm{Y}$ & $\mathrm{Z}$ \\
\hline 1 & 6 & 0 & -1712027 & -1033502 & -0.142959 \\
\hline 1 & 6 & & & & \\
\hline 2 & 6 & 0 & -2.052000 & 0.466578 & 0.126979 \\
\hline 3 & 6 & 0 & -0.915044 & 1.432635 & -0.303719 \\
\hline 4 & 6 & 0 & 1.921645 & 0.929432 & -0.078857 \\
\hline 5 & 6 & 0 & 2.105979 & -0.619870 & 0.049627 \\
\hline 6 & 6 & 0 & 0.753523 & -1.097961 & -0.420308 \\
\hline 7 & 6 & 0 & -0.321565 & -1.303045 & 0.364599 \\
\hline 8 & 1 & 0 & 0.324983 & 1.070026 & 1.680868 \\
\hline 9 & 1 & 0 & -0.794283 & 1.413727 & -1.396316 \\
\hline 10 & 1 & 0 & -0.191379 & -1.439575 & 1.437970 \\
\hline 11 & 1 & 0 & 0.573924 & -0.966784 & -1.489829 \\
\hline 12 & 1 & 0 & -1.234949 & 2.456092 & -0.047992 \\
\hline 13 & 1 & 0 & -2.464462 & -1.671512 & 0.334565 \\
\hline 14 & 1 & 0 & -2.992063 & 0.713345 & -0.383348 \\
\hline 15 & 1 & 0 & 2.940912 & -1.002076 & -0.548506 \\
\hline 16 & 1 & 0 & 2.685161 & 1.469534 & 0.495501 \\
\hline 17 & 1 & 0 & -2.233998 & 0.594914 & 1.201867 \\
\hline 18 & 1 & 0 & 2.275986 & -0.898207 & 1.095570 \\
\hline 19 & 1 & 0 & -1.756187 & -1.223216 & -1.223010 \\
\hline 20 & 1 & 0 & 2.031302 & 1.215461 & -1.133228 \\
\hline 21 & 5 & 0 & 0.430398 & 1.124533 & 0.480745 \\
\hline
\end{tabular}

